\title{
Improved clinical outcomes of rhG-CSF-mobilized blood and marrow haploidentical transplantation compared to propensity score-matched rhG-CSF-primed peripheral blood stem cell haploidentical transplantation: a multicenter study
}

\author{
Xiangyu Zhao ${ }^{1 \dagger}$, Feng Gao ${ }^{2 \dagger}$, Xiaohui Zhang ${ }^{1 \dagger}$, Yu Wang ${ }^{1,3}$, Lanping Xu${ }^{1}$, Kaiyan Liu ${ }^{1}$, \\ Xiaosu Zhao ${ }^{1,3}$, Yingjun Chang ${ }^{1,3}$, Han Wei ${ }^{1}$, Huan Chen ${ }^{1}$, Yuhong Chen ${ }^{1}$, Zhengfan Jiang ${ }^{4,5}$ \& \\ Xiaojun Huang ${ }^{1,3,5^{*}}$ \\ ${ }^{1}$ Peking University People’s Hospital \& Institute of Hematology, Beijing Key Laboratory of Hematopoietic Stem Cell Transplantation, \\ Beijing 100044, China; \\ ${ }^{2}$ North China University of Science and Technology Affiliated Hospital, Tangshan 063000, China: \\ ${ }^{3}$ Peking University Qinghe Hospital, Beijing 100085, China; \\ ${ }^{4}$ State Key Laboratory of Protein and Plant Gene Research, Key Laboratory of Cell Proliferation and Differentiation of the Ministry of \\ Education, School of Life Sciences, Peking University, Beijing 100871, China; \\ ${ }^{5}$ Peking-Tsinghua Center for Life Sciences, Beijing 100084, China
}

Received April 13,2016; accepted May 26,2016; published online August 15, 2016

\begin{abstract}
The effects of haploidentical rhG-CSF-mobilized blood and marrow transplantation (HBMT) on hematological malignances are well established. Previous prospective single-center studies have demonstrated better survival after HBMT versus haploidentical rhG-CSF-mobilized peripheral blood stem cell transplantation (HPBSCT) for acute leukemia (AL) not in remission (NR) or in more than the second complete remission ( $>$ CR2). To test the hypothesis that HBMT is still superior to HPBSCT for patients with AL, multiple myeloma (MM), or non-Hodgkin lymphoma (NHL) in CR1/CR2 and for patients with chronic myeloid leukemia in the first and second chronic phase lacking a matched donor, we designed a propensity score method-based multicenter study. Hematopoietic recovery, acute graft-versus-host disease (aGVHD), and chronic GVHD were comparable between the HBMT group $(n=168)$ and the HPBSCT group $(n=42)$. No significant differences were found in non-relapse mortality rate $(20.17 \% \pm 3.58 \%$ and $27.24 \% \pm 7.16 \%, P=0.18)$ or relapse rate $(19.96 \% \pm 3.72 \%$ and $28.49 \% \pm 8.25 \%, P=0.32)$ between the HBMT group and the HPBSCT group. HBMT recipients had better overall survival $(65.0 \% \pm 4.2 \%$ and $54.2 \% \pm 8.3 \%, P=0.037)$ and disease-free survival $(59.9 \% \pm 4.6 \%$ and $44.3 \% \pm 8.7 \%, P=0.051)$. Multivariate analysis showed that HPBSCT was associated with poorer DFS (HR $(95 \% \mathrm{CI}), 1.639$ (0.995-2.699), $P=0.052)$. Our comparisons showed that HBMT was superior to HPBSCT as a post-remission treatment for patients lacking an identical donor.
\end{abstract}

HBMT, GPB, Beijing Protocol, HSCT

Citation: Zhao, X., Gao, F., Zhang, X., Wang, Y., Xu, L., Liu, K., Zhao, X., Chang, Y., Wei, H., Chen, H., Chen, Y., Jiang, Z., and Huang, X. (2016). Improved clinical outcomes of rhG-CSF-mobilized blood and marrow haploidentical transplantation compared to propensity score-matched rhG-CSF-primed peripheral blood stem cell haploidentical transplantation: a multicenter study. Sci China Life Sci 59, 1139-1148. doi: 10.1007/s11427-016-0014-8

$\dagger$ Contributed equally to this work

*Corresponding author (email: huangxiaojun@bjmu.edu.cn) 


\section{INTRODUCTION}

Allogeneic hematopoietic stem cell transplantation (allo-HSCT) is a potentially curative treatment for hematological malignancies and blood diseases. With advancements in the field of haploidentical allo-HSCT (haplo-HSCT) in the last decade, including a new transplantation model (Apperley et al., 2016; Aversa et al., 2005; Bashey et al., 2013; Di Bartolomeo et al., 2013; Huang et al., 2006; Raiola et al., 2013; Solomon et al., 2012), haplo-HSCT would be practical for patients lacking an HLA-matched sibling or unrelated donor. To perform HSC grafts for haplo-HSCT, some centers use bone marrow (BM) grafts, and some centers use G-CSF-mobilized BM (GBM) or G-CSF-mobilized PBSCs (GPBs). Over the past 15 years, by using a combination of GBM and GPBs, as well as antithymocyte globulin (ATG) administration for the prophylaxis of graft-versus-host disease (GVHD) and graft rejection, the Beijing group initiated one of the earliest clinical trials to explore unmanipulated myeloablative haploidentical blood and marrow transplantation (HBMT) for leukemia (Huang et al., 2006; Lv and Huang, 2015). However, which type of grafts would be best for haplo-HSCT is still unknown. The use of GBM grafts in comparison with BM or GPB grafts in allo-HSCT from HLA identical sibling donors (ISDs) is either better or similar (Chang and Huang, 2011; Pessach et al., 2015). The use of the combination of GBM and GPB grafts in comparison with GPB grafts in allo-HSCT from ISDs demonstrated that GVHD incidence was significantly lower, and neutrophil and platelet engraftment were faster, making overall survival (OS) superior in the group with a combination of GBM and GPB (Zhao et al., 2013). However, the above results obtained with transplants from HLA-identical siblings or unrelated donors may not be applicable to transplants from haploidentical donors, given the greater genetic diversity in the haploidentical recipient.

Our previous single-center studies have demonstrated better survival after HBMT compared to haploidentical GPB allo-HSCT (HPBSCT) for high-risk acute leukemia (AL) (not in remission (NR) or in more than the second complete remission (>CR2)) (Xu et al., 2010). Which procedure is the optimal HSC graft for standard-risk hematological malignances (AL, multiple myeloma (MM), and non-Hodgkin lymphomas (NHL) in CR1/CR2 and patients with chronic myeloid leukemia (CML) in the first and second chronic phase $(\mathrm{CP} 1 / \mathrm{CP} 2))$ remains unknown. It would be hard to perform a randomized study on standard-risk patients using haplo-HSCT. Recently, the propensity score approach has been widely used, and it has been proposed to mimic a clinical trial. The propensity score approach can be used to produce an unbiased comparison of the treatment effect under some nonrandomized conditions. To test the hypothesis that HBMT is superior to HPBSCT as a post-remission therapy for patients with standard-risk hematological malignances who lack a matched donor, we designed a multicenter study comparing HPBSCT vs. propensity score method-matched HBMT. Our comparisons showed that HBMT was superior as a post-remission treatment for hematological malignances in patients lacking an identical donor.

\section{RESULTS}

\section{Neutrophil and platelet engraftment}

The neutrophil engraftments occurred at 13 days (range, 8-32 days) for the HBMT recipients and at 13 days (range, 9-25 days) for the HPBSCT recipients. The 100-day cumulative incidence $( \pm \mathrm{SE})$ of neutrophil engraftment in the haplo-HSCT group was comparable (HBMT group, $99.4 \% \pm 0.06 \%$; HPBSCT group, $92.9 \% \pm 4.0 \%, P=0.328)$. The platelet engraftments occurred at 22 days (range, 7-220 days) and 16 days (range, 8-40 days) for the HBMT and HPBSCT recipients, respectively. The 100-day cumulative incidence $( \pm \mathrm{SE})$ of platelet engraftment in the HBMT group was comparable to the HPBSCT group (HBMT group, $92.86 \% \pm 2.29 \%$; HPBSCT group, $90.48 \% \pm 4.89 \%, P=0.74)$.

\section{GVHD}

The 100-day cumulative incidence $( \pm \mathrm{SE})$ of grade I-IV acute GVHD (aGVHD), grade II-IV aGVHD and grade III-IV aGVHD was comparable between the two groups (I-IV aGVHD, HBMT group: $58.33 \% \pm 3.82 \%$ vs. HPBSCT group: $45.24 \% \pm 7.81 \%, P=0.22$; II-IV aGVHD, HBMT group: $33.33 \% \pm 3.7 \%$ vs. HPBSCT group: $28.57 \% \pm 7.07 \%, P=0.71$; III-IV aGVHD, HBMT group: $7.74 \% \pm 2.07 \%$ vs. HPBSCT group: $2.38 \% \pm 2.38 \%, P=0.21$; Figure $1 \mathrm{~A}-\mathrm{C}$, respectively).

A total of 158 patients in the HBMT group and 38 patients in the HPBSCT group survived longer than 100 days after HSCT and suffered from chronic GVHD. The cumulative incidence $( \pm \mathrm{SE})$ of total chronic GVHD or extensive chronic GVHD in the HBMT group was comparable to that in the HPBSCT group (total chronic GVHD: HBMT group, $50.83 \% \pm 4.01 \%$, HPBSCT group, $56.14 \% \pm 8.41 \%$, $P=0.62$, Figure 1D; extensive chronic GVHD: HBMT group, $19.11 \% \pm 3.15 \%$, HPBSCT group, $13.38 \% \pm 5.69 \%, P=0.42$, Figure 1E).

\section{Cytomegalovirus (CMV), Epstein-Barr virus (EBV) and invasive fungal infection (IFI)}

At day 100 after HSCT, the cumulative incidence $( \pm \mathrm{SE})$ of $\mathrm{CMV}$ antigenemia was $66.02 \% \pm 3.82 \%$ vs. $73.81 \% \pm 7.0 \%$ for the HBMT group versus the HPBSCT group $(P=0.49)$ (Figure 2A). The cumulative incidence $( \pm \mathrm{SE})$ of CMV-related disease was comparable between the two groups (HBMT group, $2.56 \% \pm 1.27 \%$, HPBSCT group, $7.14 \% \pm 4.0 \%, P=0.24)$.

At day 100 after HSCT, the cumulative incidence $( \pm \mathrm{SE})$ of EBV viremia was $8.3 \% \pm 2.5 \%$ and $2.5 \% \pm 2.5 \%$ in the 

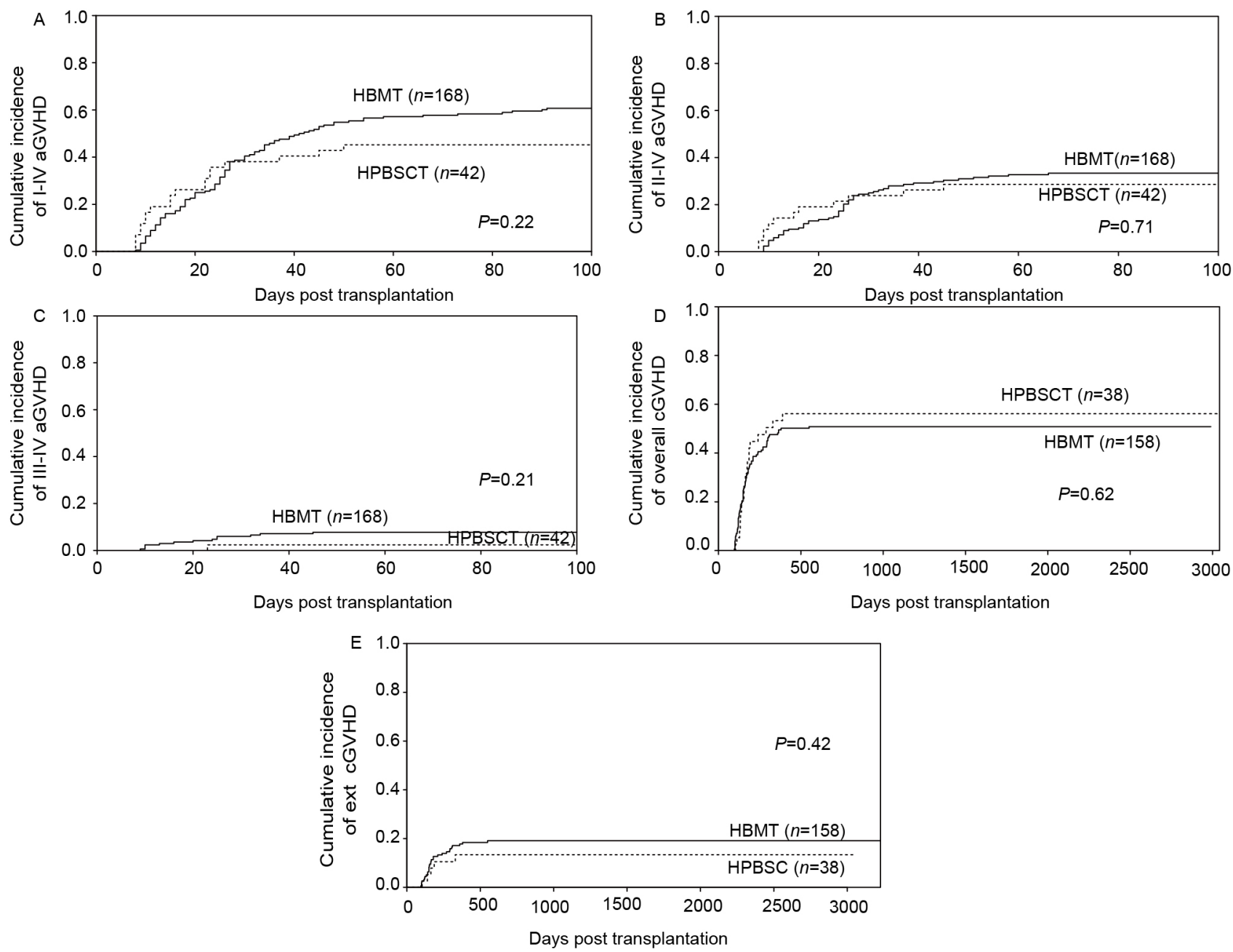

Figure 1 The acute and chronic GVHD occurrence between the HBMT group and the HPBSCT group. A, Grade I-IV acute GVHD; B, grade II-IV acute GVHD; C, grade III-IV acute GVHD; D, overall chronic GVHD; E, extensive chronic GVHD.
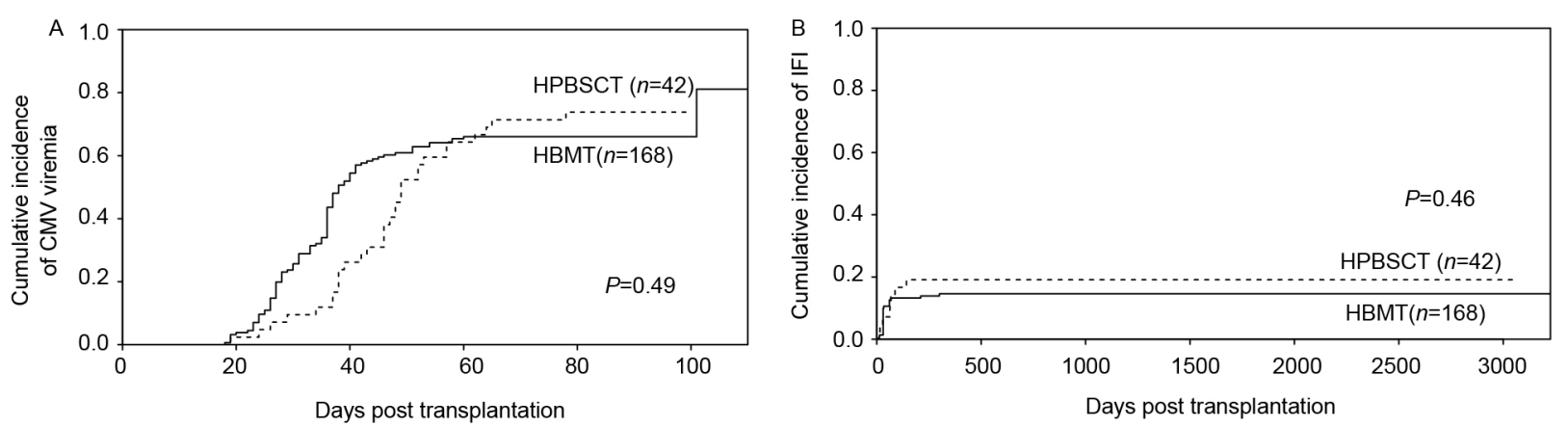

Figure 2 Infection with CMV and invasive fungi (IFI) between the HBMT group and the HPBSCT group. A, CMV viremia; B, invasive fungal infection (IFI).

HBMT and HPBSCT groups, respectively $(P=0.18)$, and the cumulative incidence of EBV-associated post-transplant lymphoproliferative disorder (PTLD) was $2.38 \% \pm 1.18 \%$ vs. $2.45 \% \pm 2.45 \%$ in the HBMT group versus the HPBSCT group $(P=0.99)$. The cumulative incidence $( \pm \mathrm{SE})$ of IFI was $14.58 \% \pm 2.88 \%$ vs. $19.12 \% \pm 6.17 \%$ in the HBMT group versus the HPBSCT group, respectively $(P=0.46$, Figure 2B).

\section{Relapse and non-relapse mortality (NRM)}

The cumulative incidence ( \pm SE) of relapse for the HBMT and HPBSCT groups was $19.96 \% \pm 3.72 \%$ and $28.49 \% \pm 8.25 \%$, respectively $(P=0.32)$ (Figure $3 \mathrm{~A})$. At the time of last follow-up, 39 patients were in relapse, and 30 patients had died of relapse, including 23 in the HBMT group and seven in the HPBSCT group, with a median time to death of 12.7 months (range, 3.7-36.9) and 10.7 months (range, 4.73-16.8) 

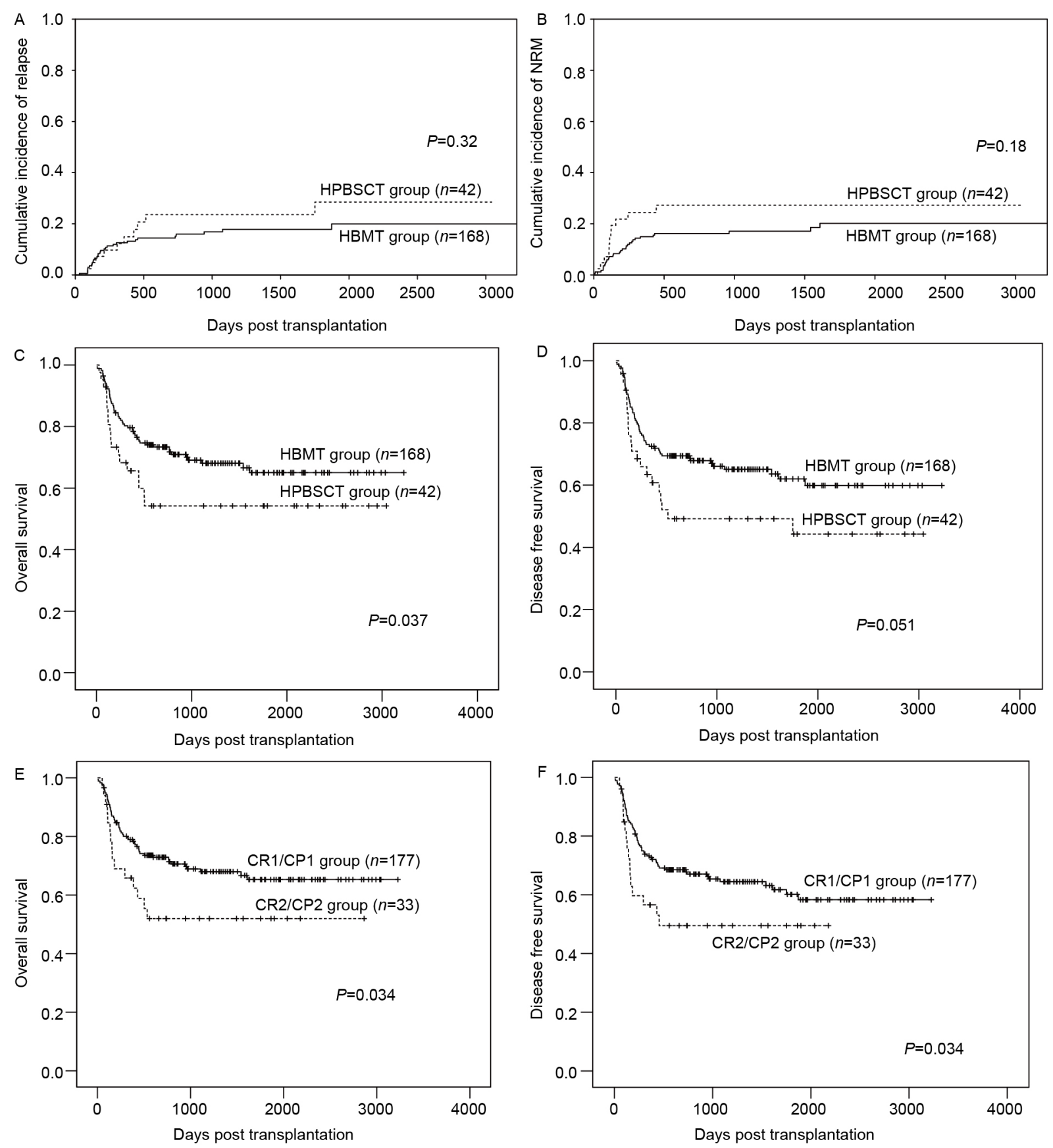

Figure 3 Clinical outcomes between the HBMT group and the HPBSCT group. A, Relapse; B, non-relapse mortality; C, overall survival; D, disease-free survival; E, overall survival; F, disease-free survival.

after HSCT, respectively. The cumulative incidence $( \pm \mathrm{SE})$ of NRM for the HBMT and HPBSCT groups was $20.17 \% \pm 3.6 \%$ and $27.24 \% \pm 7.16 \%$, respectively $(P=0.18)$ (Figure $3 \mathrm{~B}$ ).

\section{Overall survival (OS) and disease-free survival (DFS)}

The percentages $( \pm$ SE) of OS in the HBMT and HPBSCT groups were $65.0 \% \pm 4.2 \%$ and $54.2 \% \pm 8.3 \%$, respectively $(P=0.037)$ (Figure $3 \mathrm{C})$. The probabilities $( \pm \mathrm{SE})$ of DFS in the HBMT and HPBSCT groups were $59.9 \% \pm 4.6 \%$ and $44.3 \% \pm 8.7 \%$, respectively $(P=0.051)$ (Figure 3D). The probabilities of OS and DFS in the CR1/CP1 group were significantly higher than those in the CR2/CP2 group (OS, $65.3 \% \pm 4.0 \%$ and $52.0 \% \pm 9.0 \%$, respectively, $P=0.034$; DFS, $58.3 \% \pm 4.5 \%$ and $49.5 \% \pm 9.0 \%$, respectively, $P=0.034$, Figure $3 \mathrm{E}$ and F). Risk factor analysis is shown in Table 1. CR2/CP2 was associated with poorer OS (HR 1.759, 95\%CI 0.993-3.116, $P=0.053)$, and HPBSCT was associated with poorer DFS (HR 1.639, 95\%CI 0.995-2.699, $P=0.052$ ). The median follow-up of survivors was 1,304 days (range; 202-3,226 days). 

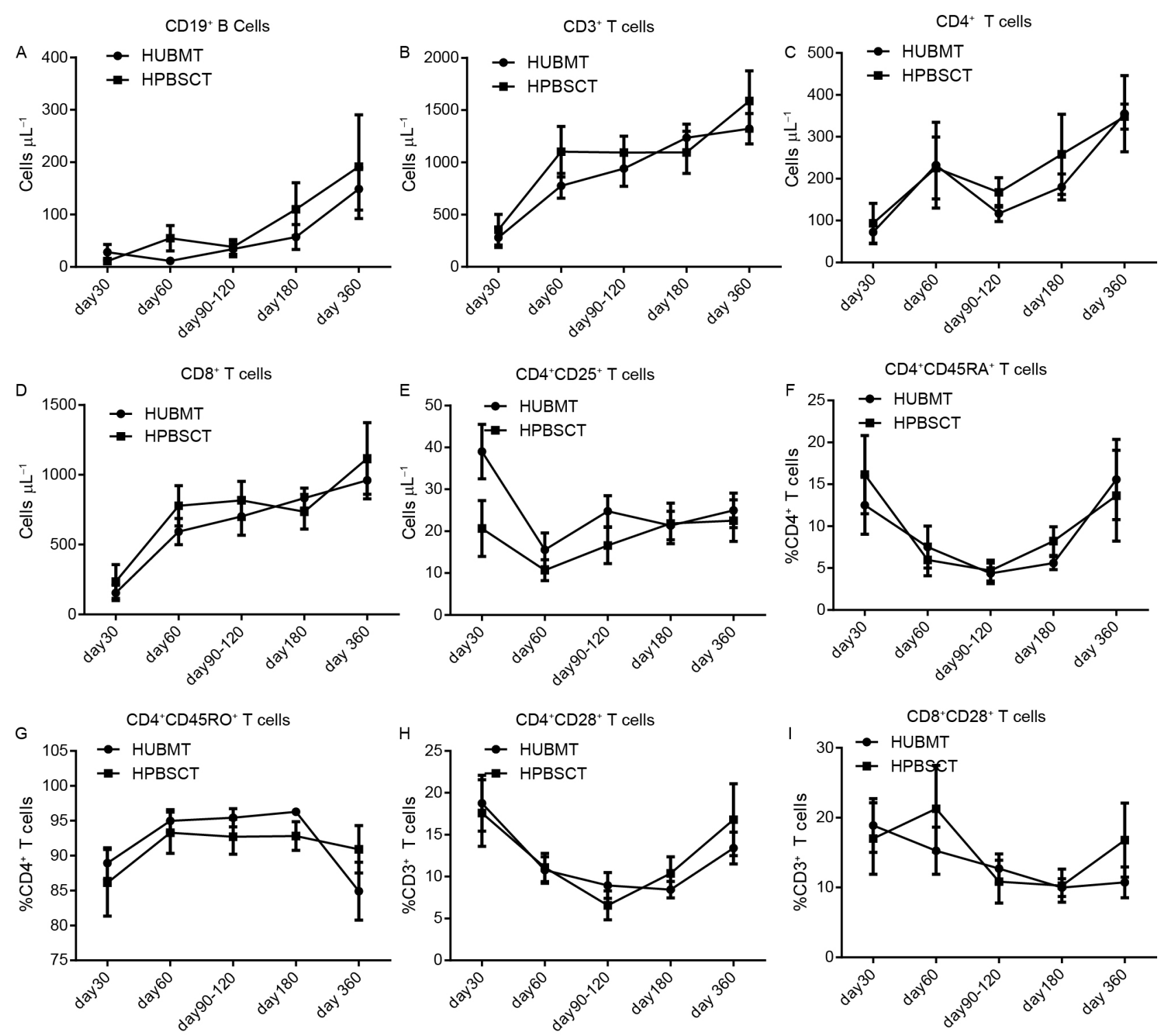

Figure 4 Immune reconstitution between the HBMT group and the HPBSCT group. Absolute numbers of (A) $\mathrm{CD} 19^{+} \mathrm{B}$ cells, (B) $\mathrm{CD} 3^{+} \mathrm{T}$ cells, (C) $\mathrm{CD} 4^{+} \mathrm{T}$ cells, and (D) $\mathrm{CD}^{+} \mathrm{T}$ cells; the percentage of (E) $\mathrm{CD}^{+} \mathrm{CD} 25^{+} \mathrm{T}$ cells, (F) $\mathrm{CD}^{+} \mathrm{CD} 45 \mathrm{RA}^{+} \mathrm{T}$ cells, and (G) $\mathrm{CD}^{+} \mathrm{CD}^{+} 5 \mathrm{RO}^{+} \mathrm{T}$ cells among the $\mathrm{CD} 3^{+} \mathrm{T}$ cells; the percentage of $(\mathrm{H}) \mathrm{CD} 4^{+} \mathrm{CD} 28^{+} \mathrm{T}$ cells among the $\mathrm{CD} 4^{+} \mathrm{T}$ cells; the percentage of (I) $\mathrm{CD} 8^{+} \mathrm{CD} 28^{+} \mathrm{T}$ cells among the $\mathrm{CD} 8^{+} \mathrm{T}$ cells.

Table 1 Multivariate analysis

\begin{tabular}{ccc}
\hline Outcome and factors & HR $(95 \% \mathrm{CI})$ & $P$-value \\
\hline OS & & \\
$\operatorname{risk}(\mathrm{CR} 2 / \mathrm{CP} 2$ vs. CR1/CP1 $)$ & $1.759(0.993-3.116)$ & 0.053 \\
DFS & $1.639(0.995-2.699)$ & 0.052 \\
\hline HPBSCT vs. HBMT & &
\end{tabular}

The percentages $( \pm \mathrm{SE})$ of OS in the HBMT and HPBSCT groups for CR1/CP1 patients were $67.1 \% \pm 4.6 \%$ and $55.9 \% \pm 8.6 \%(P=0.023)$, respectively, and for CR2/CP2 patients they were $53.8 \% \pm 9.8 \%$ and $33.3 \% \pm 22.5 \%(P=0.895)$, respectively. The percentages $( \pm \mathrm{SE})$ of DFS in the HBMT and HPBSCT groups for CR1/CP1 patients were $61.8 \% \pm 5.1 \%$ and $45.1 \% \pm 9.1 \% \quad(P=0.020)$, respectively, and for $\mathrm{CR} 2 / \mathrm{CP} 2$ patients they were $50.0 \% \pm 9.8 \%$ and $33.3 \% \pm 25.5 \%(P=0.894)$, respectively.

\section{Immune reconstitution}

Immune recovery after transplantation was assessed between January 2006 and May 2008 on days 30, 60, 90-120, 180, and 360 based on lymphocyte immunophenotyping. There were a total of 13 patients in the HPBSCT group and 19 matched patients in the HBMT group with complete sets of immunological data. As shown in Figure 4, there were no differences in the following measurements between the two groups: the absolute number of the $\mathrm{CD}^{+}, \mathrm{CD}^{+}$or $\mathrm{CD}^{+} \mathrm{T}$ cells; the percentage of $\mathrm{CD} 4^{-} \mathrm{CD} 8^{-}, \mathrm{CD}^{+} \mathrm{CD} 28^{+}$, or $\mathrm{CD} 4^{+} \mathrm{CD} 28^{+}$cells among the $\mathrm{CD}^{+} \mathrm{T}$ cells; or the percentage of $\mathrm{CD} 4^{+} \mathrm{CD} 45 \mathrm{RA}^{+}$ or $\mathrm{CD}^{+} \mathrm{CD}^{4} 5 \mathrm{RO}^{+}$cells among the $\mathrm{CD}^{+} \mathrm{T}$ cells. However, 
the absolute number of $\mathrm{CD} 19^{+}$cells was higher in the HPBSCT group at day 60 compared to the HBMT group $(P=0.04$, Figure $4 \mathrm{~A}$ ). The expression of $\mathrm{CD}^{2} 5^{+}$on $\mathrm{CD}^{+}$cells had a trend toward being higher in the HBMT group at day $30 \mathrm{com}-$ pared to the HPBSCT group (Figure 4E, $P=0.087$ ).

\section{DISCUSSION}

This multicenter, propensity score-matched study showed a significant survival difference between transplant recipients who received GPB and those who received the mixture of GBM and GPB from haploidentical donors. The results were similar among recipients with $\mathrm{CR} 1 / \mathrm{CP} 1$ or $\mathrm{CR} 2 / \mathrm{CP} 2$, although the lack of significant differences in CR2/CP2 patients was probably due to the limited number of patients. Other transplantation outcomes, including acute GVHD, chronic GVHD, CMV viremia, CMV disease, EBV viremia, IFI, relapse rates, and non-relapse mortality were comparable in the HBMT group compared to the HPBSCT group.

This is the first study to compare the clinical outcomes of standard-risk patients who underwent HBMT with those who underwent HPBSCT. We focused on standard-risk patients because they should be more comparable in terms of recipient characteristics between the HBMT and HPBSCT groups, in comparison to previous retrospective cohort studies. Meanwhile, all the patients underwent a uniform conditioning regimen as well as GVHD prophylaxis, except for the different stem cell source. Therefore, for all standard-risk patients, the use of the mixture of GBM and GPBs should increase the length of overall survival.

In accordance with our previous report (Xu et al., 2010; Zhao et al., 2013), there were trends of lower NRM and relapse rate in the GBM/GPB mixed stem cell source group than in the GPB graft group. Therefore, the GBM/GPB mixed harvest was superior to GPB in terms of overall survival in standard-risk patients. Meanwhile, although there was no significant difference, the HBMT group tended to have faster neutrophil engraftment than the HPBSCT group, in accordance with our previous report (Xu et al., 2010) (Zhao et al., 2013).

In accordance with HLA-matched ISDs (Zhao et al., 2013), acute GVHD was comparable between the HBMT group and the HPBSCT group in this study of standard-risk post-haplo-HSCT patients. However, one study reported more grade II-IV acute GVHD in the HPBSCT group than in the HBMT group in high-risk patients after haplo-HSCT (Xu et al., 2010). Based on our previous study using our haploidentical transplantation, matching the relationship of patients to donors (mother donors are associated with higher GVHD) and donor characteristics should influence the GVHD occurrence (Wang et al., 2014a, b). In this study, donor-patient relationships were comparable between groups. However, because of the small sample sizes, there have been significant differences in donor-patient relation- ships as well as the number of HLA locus mismatches in the previous high-risk cohort, which could affect the acute GVHD occurrence (Xu et al., 2010).

No significant differences were found in immune reconstitution between the HBMT group and the HPBSCT group in this study, except that the HPBSCT group had faster CD19 ${ }^{+}$ B cell recovery at day 60 , and the HBMT group had a higher frequency of $\mathrm{CD}^{+} \mathrm{CD} 25^{+} \mathrm{T}$ cells at day 30 . It would be hard to clarify their clear clinical significance based on our small sample size. Further studies should be performed on the relationship between stem cell source composition and immune reconstitution.

Only two retrospective studies have explored the effect of GPB graft or BM graft on haploidentical transplantation. Bradstock et al. compared outcomes for two retrospective cohorts of patients undergoing reduced-intensity conditioning therapy transplantation from haploidentical related donors and receiving post-transplant prophylaxis against GVHD with high-dose cyclophosphamide (Cy), tacrolimus, and mycophenolate. They demonstrated that the use of GPBs for haploidentical RIC transplants is a feasible strategy with equivalent rates of acute and chronic GVHD, equivalent risk of relapse, and lower non-relapse mortality compared to BM (Bradstock et al., 2015). Castagna et al. also retrospectively analyzed the use of BM versus GPBs in a cohort of patients receiving haploidentical $\mathrm{T}$ cell-replete transplantation after a non-myeloablative conditioning regimen with post-infusion Cy. They found that the engraftment, GVHD, relapse, NRM and survival were similar in the two groups. Therefore, GPBs could be a valid alternative to BM after transplantation from a haploidentical donor using post-infusion Cy (Castagna et al., 2014).

Many approaches have been developed or are currently being designed to improve transplant outcomes of HBMT, such as investigating the optimal dose of ATG (Wang et al., 2014b), selecting the best donor (Wang et al., 2014a) and using mDLI (Huang et al., 2007, 2008a, b, 2009; Wang et al., 2012) to reduce relapse. Therefore, the HBMT protocol has been one of the most commonly used haploidentical transplant protocols and has come to be known as the "Beijing protocol" (Apperley et al., 2016; Wang et al., 2013). It offers rapid immune recovery (Chang et al., 2014), desirable health-related quality of life and comparable survival rate to HLA-identical sibling transplantation (Mo et al., 2012, 2013) or HLA-matched unrelated donor transplantation (Xiao-Jun et al., 2009). The Beijing protocol also shows superiority in treating pediatric hematological malignancies compared with umbilical cord blood transplantation and could be successfully used as a post-remission treatment algorithm for adults with acute myeloid leukemia and unfavorable cytogenetics (Mo et al., 2014). Based on the current study, as well as our previous study (Xu et al., 2010), GBM/GPB mixed stem cells show superiority in overall survival compared to GPB 
stem cells for haplo-HSCT, suggesting that the combination of GBM and GPB as the source of stem cells contributes to the clinical efficacy of the Beijing Protocol.

A limitation of our study could be that, even though well matched, the groups were not well balanced for number of patients (168 vs. 42) and that this prevented us from reaching significance in the statistical analysis of NRM and relapse. In fact, NRM and relapse incidence were slightly higher in HPBSCT (cumulative incidence of relapse: HPBSCT 28\% vs. HBMT 19.9\%; NRM: HPBSCT 27\% vs. HBMT 20.1\%), although not significantly. Meanwhile, donor preference may affect the choice of stem cell source. Donors who declined to donate bone marrow were put into the HPBSCT group. Therefore, donor preference was a bias of this study, although we used the propensity score-matched patients' characteristics and multiple centers to keep this bias to a minimum. We need to perform a well-designed, prospective, randomized trial in the future to confirm this conclusion.

In conclusion, our comparisons showed that HBMT was superior to HPBSCT as a post-remission treatment for patients lacking an identical donor. The encouraging overall survival experienced in the HBMT cohort provides a rationale for further exploring this strategy in future prospective clinical trials to collect data from the national bone marrow registration of China and to compare the stem cell source (GBM/GPB mixed stem cells or just GPB stem cells) in recipients of transplants from haploidentical donors.

\section{MATERIALS AND METHODS}

\section{Patients}

Eligible patients were those who lacked a suitable matched related donor. A total of 1,488 patients with AML, ALL, CML, MM, or NHL receiving either a HBMT $(n=1,431)$ or a HPBSCT from a family donor $(n=57)$ between January 2002 and December 2014 from three centers in China were enrolled. We performed a matched-pair comparison of the HBMT group with a subset of closely matched HPBSCT patients selected based on propensity score matching. The clinical outcomes were compared in HBMT $(n=168)$ and HPBSCT patients $(n=42)$ after they were matched using propensity score analysis. Table 2 summarizes the demographic and clinical characteristics of the standard-risk patients after propensity score-matched analysis in this study. Patients' clinical outcomes were reported to the Peking University Institute of Hematology by the three centers. Informed consent was obtained from all of the patients. The study protocol was approved by the ethics committee of Peking University People's Hospital.

\section{Transplant regimen}

The major preconditioning consisted of cytarabine $\left(4 \mathrm{~g} \mathrm{~m}^{-2}\right.$ day $^{-1},-10$ to -9 days), busulfan (BU, $4 \mathrm{mg} \mathrm{kg}^{-1}$ day $^{-1}$ admin- istered orally on days -8 to -6 before January 2008 or $3.2 \mathrm{mg}$ $\mathrm{kg}^{-1}$ day $^{-1}$ administered intravenously on days -8 to -6 after January 2008), cyclophosphamide (CY, $1.8 \mathrm{~g} \mathrm{~m}^{-2}$ day $^{-1},-5$ to -4 days), simustine ( $250 \mathrm{mg} \mathrm{m}^{-2},-3$ days), and rabbit antithymocyte globulin (ATG; thymoglobulin, $2.5 \mathrm{mg} \mathrm{kg}^{-1},-5$ to -2 days; Sanofi, France). All of the haplo-HSCT recipients received cyclosporine $\mathrm{A}$, mycophenolate mofetil, and short-term methotrexate for GVHD prophylaxis. The criteria for choosing HBMT or HPBSCT were based on local protocol and donor preference. HBMT was suggested by the local protocol, but if the donors declined to donate bone marrow, the patients were put into the HPBSCT group. The prevention and surveillance of infections at our institute have previously been described in detail (Huang et al., 2006). The diagnosis of GVHD was in accordance with the common international criteria (Filipovich et al., 2005; Przepiorka et al., 1995).

\section{HLA typing and stem cell harvesting}

HLA typing and stem cell harvesting were previously described in detail ${ }^{3}$. Donors were primed with rhG-CSF (Filgrastim, Kirin, Japan; $5 \mathrm{mg} \mathrm{kg}^{-1}$ per day) injected s.c. for five to six consecutive days. In the HPBSCT group, on the fourth and fifth day (and on the sixth day if needed), GPBs were collected using a COBE Blood Cell Separator (Spectra LRS, COBE BCT Inc., USA) at a rate of $80 \mathrm{~mL} \mathrm{~min}^{-1}$ from a total blood volume of 150-200 mL kg-1. The fresh and unmanipulated GPBs were infused into the recipient on the day of collection. All donor-recipient pairs were typed at the HLA-A, $\mathrm{B}$, and DR loci at our institute. To determine HLA-A and HLA-B status, low-resolution DNA techniques were used. High-resolution techniques were used for HLA-DRB1 typing. Each patient with haplo-HSCT received stem cells from a family member who shared the one HLA haplotype with the patient but differed to varying degrees for the HLA-A, B, and $\mathrm{D}$ antigens of the haplotype, which were not shared. In addition to typing each donor-recipient pair, HLA typing was performed on parents and offspring only for analysis, to guarantee true haploid genetic background.

\section{Definition and assessments}

Standard-risk patients were defined as patients with AML, ALL, NHL, or MM in CR1/CR2 and patients with CML in $\mathrm{CP} 1 / \mathrm{CP} 2$. Neutrophil engraftment was defined as the first day of an absolute neutrophil count (ANC) of $0.5 \times 10^{9} \mathrm{~L}^{-1}$ or more for three consecutive days, and platelet engraftment was defined as the first day of a platelet count of $20 \times 10^{9}$ $\mathrm{L}^{-1}$ or more for seven consecutive days without transfusion. Chronic GVHD was evaluated in patients who survived for more than 100 days and had a sustained engraftment. OS was defined as time from transplantation to death from any cause. DFS was defined as survival in continuous CR. Relapse was defined as morphological evidence of the disease in the peripheral blood, bone marrow, or extramedullary sites. NRM 
Table 2 Patient's characteristics

\begin{tabular}{|c|c|c|c|}
\hline & HBMT & HPBSCT & $P$-value \\
\hline Characteristic & $n=168$ & $n=42$ & \\
\hline Diagnosis-no. (\%) & & & 0.647 \\
\hline Acute myeloid leukemia (AML) & $66(39.3 \%)$ & $14(33.4 \%)$ & \\
\hline Acute lymphoblastic leukemia (ALL) & $64(38.1 \%)$ & $14(33.3 \%)$ & \\
\hline Chronic myeloid leukemia (CML) & $32(19 \%)$ & $13(31 \%)$ & \\
\hline Multiple myeloma (MM) & $1(0.6 \%)$ & $0(0 \%)$ & \\
\hline Non-Hodgkin lymphomas (NHL) & $5(3.0 \%)$ & $1(2.4 \%)$ & \\
\hline Disease risk before transplantation & & & 0.43 \\
\hline $\mathrm{CR} 1 / \mathrm{CP} 1$ & $142(84.5 \%)$ & $35(83.3 \%)$ & \\
\hline $\mathrm{CR} 2 / \mathrm{CP} 2$ & $26(15.5 \%)$ & $7(16.7 \%)$ & \\
\hline Patient age (median, range) & $26(3-57)$ & $36(28-44)$ & \\
\hline Donor age (median, range) & $33(13-54)$ & $37(26-48)$ & \\
\hline Donor-recipient relationship-no. (\%) & & & 0.652 \\
\hline Male-male & $53(32 \%)$ & $11(26 \%)$ & \\
\hline Male-female & $21(13 \%)$ & $8(19 \%)$ & \\
\hline Female-male & $60(36 \%)$ & $16(38 \%)$ & \\
\hline Female-female & $34(20 \%)$ & $7(17 \%)$ & \\
\hline Donor-recipient relationship-no. (\%) & & & 0.584 \\
\hline Father-child & $42(25 \%)$ & $5(11.9 \%)$ & \\
\hline Mother-child & $35(20.8 \%)$ & $9(21.4 \%)$ & \\
\hline Child-parent & $22(13.1 \%)$ & $3(7.1 \%)$ & \\
\hline Sibling-sibling & $68(40.5 \%)$ & $23(54.8 \%)$ & \\
\hline Cousin-cousin & $5(0.6 \%)$ & $1(2.4 \%)$ & \\
\hline Anunt or uncle-nephew & $0(0 \%)$ & $1(2.4 \%)$ & \\
\hline \multicolumn{4}{|l|}{ Conditioning regimen - no. $(\%)$} \\
\hline Busulfan + cyclophosphamide & $168(100 \%)$ & $42(100 \%)$ & \\
\hline \multicolumn{4}{|l|}{ GVHD prophylaxis-no. (\%) } \\
\hline ATG + cyclosporine + methotrexate & $168(100 \%)$ & $42(100 \%)$ & \\
\hline \multicolumn{2}{|c|}{ No. of donor mismatches at HLA-A, B, and DRB1—no./total no. (\%) } & & 0.517 \\
\hline 0 & $1(0.6 \%)$ & $1(2.4 \%)$ & \\
\hline 1 & $11(6.5 \%)$ & $1(2.4 \%)$ & \\
\hline 2 & $55(32.7 \%)$ & $13(31.0 \%)$ & \\
\hline 3 & $101(60.1 \%)$ & $27(64.3 \%)$ & \\
\hline \multicolumn{4}{|l|}{$\mathrm{CD} 34^{+}$cell dose per kilogram $\left(\times 10^{6}\right)$} \\
\hline Median (range) & $2.03(0.52-9.77)$ & $2.46(1.68-3.25)$ & 0.901 \\
\hline \multicolumn{4}{|l|}{$\mathrm{CD}^{+}$cell dose per kilogram $\left(\times 10^{8}\right)$} \\
\hline Median (range) & $1.50(0.12-5.44)$ & $2.28(2.05-2.51)$ & $<0.001$ \\
\hline
\end{tabular}

was defined as death after HSCT without disease progression or relapse.

\section{Endpoints}

The primary endpoint of comparison was OS. Secondary endpoints were hematopoietic recovery, CMV, EBV, invasive fungal infection (IFI), acute GVHD (grade I-IV, II-IV and III-IV), chronic GVHD (overall and extensive), NRM, relapse and DFS.

\section{Immune reconstitution}

Flow cytometry analysis was performed using a four-color flow cytometer (Becton-Dickinson, American). Immune reconstitution was analyzed using three sets of 4-color antibody panels: CD45-PerCP, CD3-APC, CD4-FITC, CD8-PE; CD4-PerCP, CD25-PE, CD45RO-APC, CD45RA-FITC; and CD10-PE, CD19-APC, CD45-PerCP, and CD34-FITC (Becton-Dickinson, USA). Fifty thousand PBMCs were collected per sample and analyzed using Cell Quest software (Bec- 
ton-Dickinson). Fluorochrome-conjugated isotype-matched nonspecific mAbs were used as negative controls for each assay.

\section{Propensity score calculation}

We calculated the propensity score matching in $\mathrm{R}$ using the Match It package with nearest-neighbor 1-to-4 matching (R Development Core Team (2006)); R, A language and environment for statistical computing. R Foundation for Statistical Computing, Vienna, Austria. ISBN: 3-900051-07-0, URL: http://www.R-project.org). Factors included in the propensity score were as follows: patient age and donor age at HSCT as a continuous variable; patient sex and donor sex (male/female) as an indicator variable; year of transplantation as a continuous variable; performance status at transplantation as an ordinal variable; risk status (CR1/CP1, $\mathrm{CR} 2 / \mathrm{CP} 2$ ) as an indicator variable; relationship of patient to donor (father, mother, child, sibling, cousin, aunt or uncle) as an indicator variable; and diagnosis (AML, ALL, CML, NHL, MM) as an indicator variable. We utilized as many variables as possible in the propensity score to reduce or eliminate the effect of treatment selection bias.

\section{Statistical analysis}

Patient characteristics and therapeutic outcomes were compared between the HBMT and HPBSCT groups. Data were censored at the time of last available follow-up. OS and DFS were estimated using the Kaplan-Meier method. Competing risk analysis was used to calculate the cumulative incidence of engraftments, GVHD, CMV, infection with EBV or invasive fungus, relapse, and NRM using the Gray test to test for differences between the HBMT and HPBSCT groups. Death without engraftment was the competing event for engraftment, death without GVHD was the competing event for GVHD, and death without infection was the competing event for infection. For NRM, relapse was the competing event; for relapse, NRM was the competing event. The final multivariate models were built using a forward stepwise model selection approach with a 5\% significance level. Final models for each outcome were reported. The end point of the last follow-up for all survivors was May 1, 2015. Immune reconstitution was compared between the HBMT and HPBSCT groups using the Wilcoxon rank-sum test. Data were primarily analyzed using the SPSS software package (SPSS Inc., USA). The R software package (version 2.6.1; http://www.r-project.org) was used for competing risk analysis.

All authors declare no competing interests. This study conformed with the Helsinki Declaration of 1975 (as revised in 2008) concerning Human and Animal Rights, and we followed the policy concerning Informed Consent as published at Springer.com.
Compliance and ethics The author(s) declare that they have no conflict of interest.

Acknowledgements This work was supported by the National Natural Science Foundation of China (81530046, 81270644, 81230013), the Major State Basic Research Development Program of China (2013CB733700), the Collaborative Innovation Center of Hematology, Peking University, China, Beijing Talents fund (2015000021223ZK26), the Milstein Medical Asian American Partnership (MMAAP) Foundation Research Project Award in Hematology, and project TG-2015-003 supported by the Health Science Promotion Project of Beijing. The authors thank all of the core facilities at the Peking University Institute of Hematology for sample collection.

Apperley, J., Niederwieser, D., Huang, X., Nagler, A., Fuchs, E., Szer, J., and Kodera, Y. (2016). Haploidentical hematopoietic stem cell transplantation: a global overview comparing Asia, the European Union, and the United States. Biol Blood Marrow Transplant 22, 23-26.

Aversa, F. (2005). Full haplotype-mismatched hematopoietic stem-cell transplantation: a phase II study in patients with acute leukemia at high risk of relapse. J Clin Oncol 23, 3447-3454.

Bashey, A., Zhang, X., Sizemore, C.A., Manion, K., Brown, S., Holland, H.K., Morris, L.E., and Solomon, S.R. (2013). T-cell-replete HLA-haploidentical hematopoietic transplantation for hematologic malignancies using post-transplantation cyclophosphamide results in outcomes equivalent to those of contemporaneous HLA-matched related and unrelated donor transplantation. J Clin Oncol 31, 1310-1316.

Bradstock, K., Bilmon, I., Kwan, J., Blyth, E., Micklethwaite, K., Huang, G., Deren, S., Byth, K., and Gottlieb, D. (2015). Influence of stem cell source on outcomes of allogeneic reduced-intensity conditioning therapy transplants using haploidentical related donors. Biol Blood Marrow Transplant 21, 1641-1645.

Castagna, L., Crocchiolo, R., Furst, S., Bramanti, S., El Cheikh, J., Sarina, B., Granata, A., Mauro, E., Faucher, C., Mohty, B., Harbi, S., Chabannon, C., Carlo-Stella, C., Santoro, A., and Blaise, D. (2014). Bone marrow compared with peripheral blood stem cells for haploidentical transplantation with a nonmyeloablative conditioning regimen and post-transplantation cyclophosphamide. Biol Blood Marrow Transplant 20, 724-729

Chang, Y.J., and Huang, X.J. (2011). Use of G-CSF-stimulated marrow in allogeneic hematopoietic stem cell transplantation settings: a comprehensive review. Clin Transplant 25, 13-23.

Chang, Y.J., Zhao, X.Y., and Huang, X.J. (2014). Immune reconstitution after haploidentical hematopoietic stem cell transplantation. Biol Blood Marrow Transplant 20, 440-449.

Di Bartolomeo, P., Santarone, S., De Angelis, G., Picardi, A., Cudillo, L., Cerretti, R., Adorno, G., Angelini, S., Andreani, M., De Felice, L., Rapanotti, M.C., Sarmati, L., Bavaro, P., Papalinetti, G., Di Nicola, M., Papola, F., Montanari, M., Nagler, A., and Arcese, W. (2013). Haploidentical, unmanipulated, G-CSF-primed bone marrow transplantation for patients with high-risk hematologic malignancies. Blood 121, 849-857.

Filipovich, A.H., Weisdorf, D., Pavletic, S., Socie, G., Wingard, J.R., Lee, S.J., Martin, P., Chien, J., Przepiorka, D., Couriel, D., Cowen, E.W., Dinndorf, P., Farrell, A., Hartzman, R., Henslee-Downey, J., Jacobsohn, D., McDonald, G., Mittleman, B., Rizzo, J.D., Robinson, M., Schubert, M., Schultz, K., Shulman, H., Turner, M., Vogelsang, G., and Flowers, M.E.D. (2005). National Institutes of Health Consensus Development Project on criteria for clinical trials in chronic graft-versus-host disease: I. Diagnosis and Staging Working Group Report. Biol Blood Marrow Transplant 11, 945-956.

Huang, X.J., Liu, D.H., Liu, K.Y., Xu, L.P., Chen, H., and Han, W. (2007). Donor lymphocyte infusion for the treatment of leukemia relapse after HLA-mismatched/haploidentical T-cell-replete hematopoietic stem cell transplantation. Haematologica 92, 414-417. 
Huang, X.J., Liu, D.H., Liu, K.Y., Xu, L.P., Chen, H., Han, W., Chen, Y.H., Wang, J.Z., Gao, Z.Y., Zhang, Y.C., Jiang, Q., Shi, H.X., and Lu, D.P. (2006). Haploidentical hematopoietic stem cell transplantation without in vitro T-cell depletion for the treatment of hematological malignancies. Bone Marrow Transplant 38, 291-297.

Huang, X.J., Liu, D.H., Liu, K.Y., Xu, L.P., Chen, Y.H., Wang, Y., Han, W., and Chen, H. (2008a). Modified donor lymphocyte infusion after HLA-mismatched/haploidentical T cell-replete hematopoietic stem cell transplantation for prophylaxis of relapse of leukemia in patients with advanced leukemia. J Clin Immunol 28, 276-283.

Huang, X.J., Wang, Y., Liu, D.H., Xu, L.P., Chen, H., Chen, Y.H., Han, W., Shi, H.X., and Liu, K.Y. (2008b). Modified donor lymphocyte infusion (DLI) for the prophylaxis of leukemia relapse after hematopoietic stem cell transplantation in patients with advanced leukemia-feasibility and safety study. J Clin Immunol 28, 390-397.

Huang, X.J., Wang, Y., Liu, D.H., Xu, L.P., Liu, K.Y., Chen, H., Chen, Y.H., Han, W., and Shi, H.X. (2009). Administration of short-term immunosuppressive agents after DLI reduces the incidence of DLI-associated acute GVHD without influencing the GVL effect. Bone Marrow Transplant 44, 309-316.

Lv, M., and Huang, X.J. (2015). Fighting against hematological malignancy in China: from unique system to global impact. Sci China Life Sci 58, 1183-1190.

Mo, X.D., Xu, L.P., Liu, D.H., Chen, Y.H., Han, W., Zhang, X.H., Chen, H., Wang, Y., Wang, J.Z., Liu, K.Y., and Huang, X.J. (2012). Patients receiving HLA-haploidentical/partially matched related allo-HSCT can achieve desirable health-related QoL that is comparable to that of patients receiving HLA-identical sibling allo-HSCT. Bone Marrow Transplant 47, 1201-1205.

Mo, X.D., Xu, L.P., Liu, D.H., Zhang, X.H., Chen, H., Chen, Y.H., Han, W., Wang, Y., Wang, F.R., Wang, J.Z., Liu, K.Y., and Huang, X.J. (2013). Nonmalignant late effects in survivors of partially matched donor hematopoietic stem cell transplantation. Biol Blood Marrow Transplant 19, 777-783.

Mo, X.D., Zhao, X.Y., Liu, D.H., Chen, Y.H., Xu, L.P., Zhang, X.H., Chen, H., Han, W., Wang, Y., Wang, F.R., Wang, J.Z., Liu, K.Y., and Huang, X.J. (2014). Umbilical cord blood transplantation and unmanipulated haploidentical hematopoietic SCT for pediatric hematologic malignances. Bone Marrow Transplant 49, 1070-1075.

Pessach, I., Resnick, I., Shimoni, A., and Nagler, A. (2015). G-CSF-primed BM for allogeneic SCT: revisited. Bone Marrow Transplant 50, 892-898.

Przepiorka, D., Weisdorf, D., Martin, P., Klingemann, H.G., Beatty, P., Hows, J., and Thomas, E.D. (1995). 1994 Consensus Conference on Acute GVHD Grading. Bone Marrow Transplant 15, 825-828.

Raiola, A.M., Dominietto, A., Ghiso, A., Di Grazia, C., Lamparelli, T., Gualandi, F., Bregante, S., Van Lint, M.T., Geroldi, S., Luchetti, S., Ballerini, F., Miglino, M., Varaldo, R., and Bacigalupo, A. (2013). Unmanipulated haploidentical bone marrow transplantation and posttransplantation cyclophosphamide for hematologic malignancies after myeloablative conditioning. Biol Blood Marrow Transplant 19, 117-122.

Solomon, S.R., Sizemore, C.A., Sanacore, M., Zhang, X., Brown, S., Holland, H.K., Morris, L.E., and Bashey, A. (2012). Haploidentical transplantation using $\mathrm{T}$ cell replete peripheral blood stem cells and myeloablative conditioning in patients with high-risk hematologic malignancies who lack conventional donors is well tolerated and produces excellent relapse-free survival: results of a prospective phase II trial. Biol Blood Marrow Transplant 18, 1859-1866.

Wang, Y., Chang, Y.J., Xu, L.P., Liu, K.Y., Liu, D.H., Zhang, X.H., Chen, H., Han, W., Chen, Y.H., Wang, F.R., Wang, J.Z., Chen, Y., Yan, C.H., Huo, M.R., Li, D., and Huang, X.J. (2014a). Who is the best donor for a related HLA haplotype-mismatched transplant?. Blood 124, 843-850.

Wang, Y., Fu, H.X., Liu, D.H., Xu, L.P., Zhang, X.H., Chang, Y.J., Chen, Y.H., Wang, F.R., Sun, Y.Q., Tang, F.F., Liu, K.Y., and Huang, X.J. (2014b). Influence of two different doses of antithymocyte globulin in patients with standard-risk disease following haploidentical transplantation: a randomized trial. Bone Marrow Transplant 49, 426-433.

Wang, Y., Liu, D.H., Liu, K.Y., Xu, L.P., Zhang, X.H., Han, W., Chen, H., Chen, Y.H., Wang, F.R., Wang, J.Z., Sun, Y.Q., and Huang, X.J. (2013). Long-term follow-up of haploidentical hematopoietic stem cell transplantation without in vitro $\mathrm{T}$ cell depletion for the treatment of leukemia. Cancer 119, 978-985.

Wang, Y., Liu, D.H., Xu, L.P., Liu, K.Y., Chen, H., Zhang, X.H., Chen, Y.H., Han, W., Wang, F.R., Wang, J.Z., Yan, C.H., and Huang, X.J. (2012). Prevention of relapse using granulocyte CSF-primed PBPCs following HLA-mismatched/haploidentical, T-cell-replete hematopoietic SCT in patients with advanced-stage acute leukemia: a retrospective risk-factor analysis. Bone Marrow Transplant 47, 1099-1104.

Xiao-Jun, H., Lan-Ping, X., Kai-Yan, L., Dai-Hong, L., Yu, W., Huan, C., Yu-Hong, C., Wei, H., Jing-Zhi, W., Yao, C., Xiao-Hui, Z., Hong-Xia, S., Feng-Rong, W., and Fei-Fei, T. (2009). Partially matched related donor transplantation can achieve outcomes comparable with unrelated donor transplantation for patients with hematologic malignancies. Clin Cancer Res 15, 4777-4783.

Xu, L.P., Liu, K.Y., Liu, D.H., Chen, H., Han, W., Chen, Y.H., Wang, Y., and Huang, X.J. (2010). The inferiority of G-PB to rhG-CSF-mobilized blood and marrow grafts as a stem cell source in patients with high-risk acute leukemia who underwent unmanipulated HLA-mismatched/haploidentical transplantation: a comparative analysis. Bone Marrow Transplant 45, 985-992.

Zhao, X., Chen, Y., Zhao, X., Liu, D., Xu, L., Wang, Y., Han, W., Chen, Y., Chen, H., Zhang, X., Liu, K., and Huang, X. (2013). Improved outcomes using G-CSF-mobilized blood and bone marrow grafts as the source of stem cells compared with G-PB after HLA-identical sibling transplantation in patients with acute leukemia. Clin Transplant 27, 844-851.

Open Access This article is distributed under the terms of the Creative Commons Attribution License, which permits any use, distribution, and reprofduction in any medium, provided the original author(s) and source are credited. 